\title{
Power-Aware QoS Enhancement in Multihop DS-CDMA Visual Sensor Networks
}

\author{
A. V. Katsenou ${ }^{1}$, E. G. Datsika ${ }^{2}$, L. P. Kondi ${ }^{1}$, E. Papapetrou ${ }^{1}$, and K. E. Parsopoulos ${ }^{1}$ \\ ${ }^{1}$ Department of Computer Science, University of Ioannina, GR-45110, Ioannina, Greece \\ ${ }^{2}$ Multimedia Technology Laboratory, School of Electr.\&Comp. Engineering, National Technical University of Athens, Greece \\ ${ }^{1}$ \{akatseno, lkon, epap, kostasp\}@cs.uoi.gr, ${ }^{2}$ edatsika@medialab.ntua.gr
}

\begin{abstract}
We propose a quality-driven method for network resource allocation with transmission power control in a multihop Direct Sequence Code Division Multiple Access (DS-CDMA) Wireless Visual Sensor Network (WVSN). A multihop WVSN typically consists of source nodes that monitor different areas and relay nodes that retransmit recorded scenes. In order to achieve the best possible video quality at the receiver while consuming the least possible transmission power, we propose a joint optimization scheme that allocates the available resources among the nodes with respect to the imposed constraints. Moreover, we formulate a weighted bi-objective optimization problem and study the tradeoff between video quality and consumed transmission power. The simulation demonstrate that excessive transmission power is used when power control is omitted for a rather small quality gain for certain nodes.
\end{abstract}

Index Terms-Power Control, Quality of Service, Resource Allocation, Wireless Visual Sensor Networks, DS-CDMA.

\section{INTRODUCTION}

Wireless Visual Sensor Networks (WVSNs) are poised to enable the widespread deployment of a plethora of lifeenhancing services and applications, such as environmental monitoring, building surveillance, etc. [1]. Traditional WVSNs are organized in centralized topologies and consist of: a) lowweight, energy-constrained sensors with wireless communication capability that are equipped with video cameras, and b) a Base Station (BS) that collects the information from the visual sensors, applies channel and source decoding to the received video of each sensor and decides on the resource allocation among all network nodes. Since the transmission range of a sensor is limited, the recorded video sequences may need to be transmitted using fixed relay nodes until they reach the BS via a multihop path as depicted in Fig. 1. To this end, the network utilizes a channel-decode-and-forward protocol. In this context, a source node's transmission causes interference to the transmitting nodes that lie within its transmission range, leading to the degradation of the received video quality at the BS.

The constraints imposed on the power consumption, the delay for video delivery and the computational complexity, along with the dynamic nature of the wireless environment, render

Effort sponsored by the Air Force Office of Scientific Research, Air Force Material Command, USAF, under grant number FA8655-12-1-0001. The U.S Government is authorized to reproduce and distribute reprints for Governmental purpose notwithstanding any copyright notation thereon. the efficient design of the aforementioned system a challenging task. The problem of power efficiency, in conjunction with the required Quality of Service (QoS), has been extensively studied in recent years and various techniques have been proposed [2], [3], [4]. In [2] the joint power control and scheduling problem in wireless multihop networks is addressed with the objective of total transmission power minimization while QoS for individual sessions in terms of payload rate and bit error rate is guaranteed. Resource allocation schemes for a multi-user, multi-relay cellular cooperative communication system are studied in [3]. The authors formulate a multiobjective tradeoff scheme to provide a balance between energy efficiency and throughput in the network. In [4], recognizing the fact that power control itself cannot meet the QoS requirements, a joint channel and power allocation scheme for cognitive radio networks is proposed. This scheme is designed to maximize the overall throughput, while guaranteeing the proportional fairness and power distribution among the cognitive radio users. All of the above bi-objective problem formulations target at network-related QoS metrics optimization and not at the end-to-end quality of the delivered information.

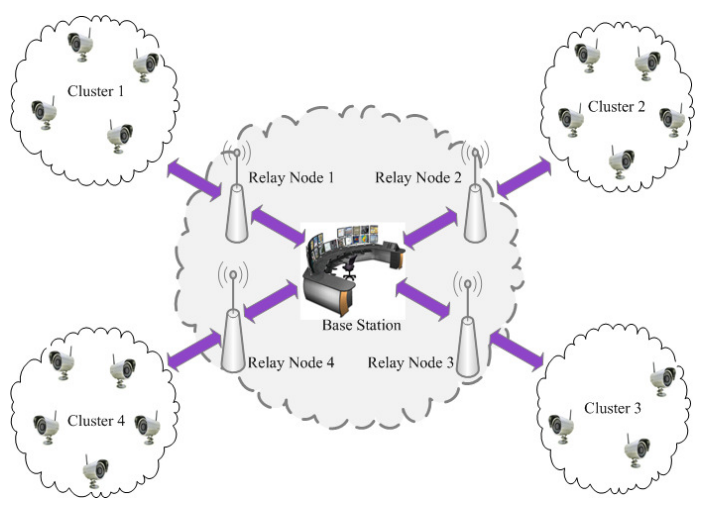

Fig. 1. Example of a centralized WVSN with two hops.

Instead of explicitly optimizing network-related parameters, such as bit error rate or throughput, this work analyzes an optimization scheme which intends to maximize the delivered video quality in terms of Peak Signal-to-Noise Ratio (PSNR) under the network's power constraints. Particularly, the network resources (transmission power, source and channel coding rates) have to be optimally allocated to the source and 
relay nodes using a quality-aware strategy, in order to maintain the end-to-end distortion at a low level for all source nodes. Moreover, power control is dictated by the battery-powered sensors.

This paper builds on the concept introduced in our previous work [5] and moves beyond by jointly considering the end-toend video quality enhancement along with the power control. For the assignment of the available resources, a compromise between two aspects is essential: on the one hand, the power consumption has to be minimized in order to prolong the WVSN's lifespan and simultaneously reduce the interference among the transmitted signals; on the other hand, the distortion of the delivered video sequences has to be minimized as well, so that the QoS requirements of an application are satisfied. Therefore, we propose a bi-objective method that jointly allocates the transmission power to the source and relay nodes, the source coding rates to the source nodes, and the channel coding rates to all nodes.

The rest of the paper is organized as follows. Section II describes the considered system model, while Section III formulates the proposed problem and method. The numerical results are presented and discussed in Section IV, and conclusions are drawn in Section V.

\section{Considered System Model}

In this work, we consider a Direct Sequence Code Division Multiple Access (DS-CDMA) based network. In such a network, each node $n$ is associated with a spreading sequence of length $L_{n}$. Furthermore, Binary Phase Shift Keying (BPSK) is used as the modulation method. Let $N$ be the number of nodes in a synchronous single-path BPSK channel, $A_{n}, b_{n}(i), \mathbf{s}_{n}$, $\mathbf{u}_{n}$ the amplitude, symbol stream, spreading code and noise of node $n$, respectively, and $\mathbf{r}(i), \mathbf{s}_{k}(i)$ and $\mathbf{u}_{n}$ vectors of length $L_{n}$. For the $i$-th bit the received signal can be expressed as:

$$
\mathbf{r}(i)=A_{1} b_{1}(i) \mathbf{s}_{1}+\sum_{n=2}^{N} A_{n} b_{n}(i) \mathbf{s}_{n}+\mathbf{u}_{n} .
$$

For a WVSN with $N$ nodes, the received power of a node $n$ is $S_{n}^{\text {rec }}=E_{n} R_{n}$ in W. $E_{n}$ is the energy-per-bit and the total transmission bit rate for source and channel coding in bits/sec is given by:

$$
R_{n}=\frac{R_{\mathrm{s}, n}}{R_{\mathrm{c}, n}}, \quad n=1,2, \ldots, N,
$$

where $R_{\mathrm{s}, n}$ is the source coding rate in bits/sec and the dimensionless number $R_{\mathrm{c}, n}$ is the channel coding rate. A node that transmits with a lower source coding rate is able to use more bits for the channel coding. It can then transmit with lower power and, as a consequence, it causes less interference to other nodes' transmissions.

Since we consider a multihop WVSN, we assume that interference exists on each link across the path to the BS from nodes that are in the effective transmission range. Similar to other approaches [6], we model interference as Additive White Gaussian Noise (AWGN). For the set $\mathbf{J}$ that consists of the interfering nodes for each hop $h$, it is assumed that $|\mathbf{J}| \leq N$. The energy-per-bit to Multiple Access Interference (MAI) and noise ratio is different in each link, depending on the nodes causing interference to the considered node $n$ and can be expressed for the $h$-th hop of a path as follows:

$$
\frac{E_{n}}{I_{0}+N_{0}}=\frac{\frac{S_{n}^{\text {rec }}}{R_{n}}}{\sum_{j=1, j \neq n}^{|\mathbf{J}|} \frac{S_{j}^{\text {rec }}}{W_{\mathrm{t}}}+N_{0}},
$$

where $I_{0} / 2$ is the two sided noise power spectral density due to MAI, $N_{0} / 2$ is the two sided noise power spectral density of background noise in $\mathrm{W} / \mathrm{Hz}, W_{\mathrm{t}}$ is the total bandwidth in $\mathrm{Hz}$ and $S_{j}^{\text {rec }}$ is the received power of node $j \in \mathbf{J}$ that causes interference to node $n$ [6]. For a given received signal power $S_{n}^{\text {rec }}$ at a distance $d$ from a node $n$, the required transmitted power $S_{n}^{\text {trans }}$ for the node $n$ can be determined by a suitable radio propagation model (as explained at the end of this Section).

A constraint imposed in the considered multihop WVSN is that each relay node $m$ needs to use a sufficient bit rate for the simultaneous forwarding of the video data, which is related to source coding rate of the related source nodes. Hence, the transmission bit rate of a relay $m$ is

$$
R_{m} \geq \frac{\sum_{z \in \mathbf{Z}} R_{\mathrm{s}, z}}{R_{\mathrm{c}, \mathrm{R}, m}}
$$

where $\mathbf{Z}$ is the set that includes the source nodes that use relay node $m$ for their data forwarding and $R_{\mathrm{c}, \mathrm{R}, m}$ is the channel coding rate for the relay node $m$.

For channel coding, we use Rate Compatible Punctured Convolutional Codes (RCPC) which map information to code bits sequentially with an encoding process that involves convolution of the useful data with a generator sequence. However, other error correction codes could be used. The Viterbi upper bound for the bit error probability $P_{b}$ is given by:

$$
P_{b} \leq \frac{1}{P} \sum_{d_{\mathrm{H}}=d_{\mathrm{free}}}^{\infty} c_{d} P_{d}
$$

where $P$ is the period of code, $P_{d}$ is the pairwise error probability in choosing between two paths of mutual Hamming distance $d_{\mathrm{H}}, d_{\text {free }}$ is the free distance of the code and $c_{d}$ is the information error weight [7]. Considering a AWGN channel with BPSK modulation, the pairwise error probability from Eq. (5) is given by:

$$
P_{d}=\operatorname{erfc}\left(\sqrt{d_{\mathrm{H}} R_{\mathrm{c}, n}\left[\frac{E_{n}}{I_{0}+N_{0}}\right]}\right)
$$

where the function $\operatorname{erfc}($.$) is the complementary error function$ given by: $\operatorname{erfc}(z)=\left(2 \int_{z}^{\infty} \exp \left(-t^{2}\right) d t\right) / \sqrt{\pi}$.

Assuming that $P b_{h, k}$ is the bit error probability for hop $h$ and the source node $k$, the end-to-end bit error probability 
across an $H$-hop path for $k$ is [8]:

$$
P b_{k}=1-\prod_{h=1}^{H}\left(1-P b_{h, k}\right) .
$$

For the compression of the recorded video sequences, the H.264/AVC standard is utilized, which offers a networkfriendly design to both real-time applications, such as video conference or surveillance applications, and nonconversational applications, like video streaming [9].

The expected distortion of a video transmitted by a specific source node depends on the bit error probability of the links across the path to the final receiver of the video. In order to calculate the expected distortion as a function of the bit error probabilities after channel decoding, we use the Universal Rate-Distortion Characteristics (URDCs) [10]. It should be noted that the errors occurring in the channel are random, thus the video distortion $D_{\mathrm{s}+\mathrm{c}, k}$ of a user $k$ is a random variable. Due to that fact we have to calculate the value of the expected distortion $E\left\{D_{\mathrm{s}+\mathrm{c}, k}\right\}$ for various realizations of the channel. The $P b$ 's needed for the URDCs are the ones we obtain after the channel decoding process.

Owing to Eq. (7), the expected end-to-end video distortion due to lossy compression and channel errors can be derived by the model for the URDC of each user $k$ used in [11], [12]:

$$
E\left\{D_{\mathrm{s}+\mathrm{c}, k}\right\}=\alpha_{k}\left[\log _{10}\left(\frac{1}{1-\prod_{h=1}^{H}\left(1-P b_{h, k}\right)}\right)\right]^{-\beta_{k}},
$$

where parameters $\alpha_{k}$ and $\beta_{k}$ are positive numbers that depend on the motion level of the transmitted video sequence and the source coding rate and may vary in time. Values of $\alpha_{k}$ for high motion video sequences are generally greater than those for low motion video sequences. These parameters are determined using mean square optimization from a few $\left(E\left\{D_{\mathrm{s}+\mathrm{c}, k}\right\}, P b_{k}\right)$ pairs. The choice of $\alpha_{k}$ and $\beta_{k}$ minimizes the square of the approximation error so that there is no need to calculate the URDCs based on simulation results for every possible value of $\mathrm{Pb}$ 's. In contrast, we compute the expected distortion for a small number of packet loss rates associated with specific $P b$ 's.

We assume clear line of sight for our model and in order to calculate the received power at a node, we employ a mixed scenario that consists of two propagation models; the Free Space (FS) and the Two Ray Ground Reflection (TRGR) models [13]. More specifically, the received power is calculated based on the FS model when the communication distance is under a threshold otherwise it is calculated based on the TRGR model, i.e.:

$$
S_{n}^{\mathrm{rec}}(d)=\left\{\begin{array}{ccc}
S_{n}^{\mathrm{trans}} \frac{G_{t} G_{r} \lambda^{2}}{(4 \pi)^{2} d^{2} l} & \text { if } d \leq d_{0} \quad \text { (FS Model) } \\
S_{n}^{\operatorname{trans}} \frac{G_{t} G_{r} h_{t}^{2} h_{r}^{2}}{d^{4} l} & \text { if } d>d_{0} & \text { (TRGR Model) }
\end{array}\right.
$$

where $d$ is the communication distance, $l \geq 1$ is the system loss factor not related to propagation, $\lambda$ the wavelength of the carrier signal, $\left(G_{t}, G_{r}\right)$ and $\left(h_{t}, h_{r}\right)$ are the antenna gain and height for the transmitter and the receiver, respectively. The distance $d_{0}$ is called cross-over distance and is calculated by $d_{0}=\left(4 \pi h_{r} h_{t} \sqrt{l}\right) / \lambda$. The used model takes advantage of the better accuracy of the TRGR model for long distances, while it avoids its poor performance for short distances.

\section{Problem Formulation and Proposed Method}

In the present paper, we propose a method that offers enhancement of the end-to-end video quality and manages the transmitted power of the WVSN nodes. Our method aims at optimally allocating the source and channel coding rates and the transmitted powers among the source nodes of a WVSN and simultaneously the necessary channel coding rates and transmitted powers to the relay nodes. For the assignment of the available resources, a compromise between the power consumption and the distortion of the delivered video sequences has to be established. Therefore, we define a bi-objective problem that actually minimizes a function of both the expected distortions of the received videos and the received powers.

We first define the following vectors for source and channel coding rates, and the received powers of source nodes $k=$ $1,2, \ldots, K$ and relay nodes $m=1,2, \ldots, M$, respectively:

$$
\begin{aligned}
& R_{\mathrm{s}}=\left(R_{\mathrm{s}, 1}, \ldots, R_{\mathrm{s}, K}\right)^{\top} ; \\
& R_{\mathrm{c}}=\left(R_{\mathrm{c}, \mathrm{S}, 1}, \ldots, R_{\mathrm{c}, \mathrm{S}, K}, R_{\mathrm{c}, \mathrm{R}, 1}, \ldots, R_{\mathrm{c}, \mathrm{R}, M}\right)^{\top} ; \\
& S^{\mathrm{rec}}=\left(S_{\mathrm{S}, 1}^{\mathrm{rec}}, \ldots, S_{\mathrm{S}, K}^{\mathrm{rec}}, S_{\mathrm{R}, 1}^{\mathrm{rec}}, \ldots, S_{\mathrm{R}, M}^{\mathrm{rec}}\right)^{\top} .
\end{aligned}
$$

For each source node $k$, the source coding rate $R_{\mathrm{s}, k}$, the channel coding rate $R_{\mathrm{c}, \mathrm{S}, k}$ and the received power $S_{\mathrm{S}, k}^{\mathrm{rec}} \in$ $\left[S_{\mathrm{S}}^{\min }, S_{\mathrm{S}}^{\max }\right]$ and for each relay node $m$ the channel coding rate $R_{\mathrm{c}, \mathrm{R}, m}$ and the received power $S_{\mathrm{R}, m}^{\mathrm{rec}} \in\left[S_{\mathrm{R}}^{\min }, S_{\mathrm{R}}^{\max }\right]$ are determined, so that the weighted aggregation of the expected video distortion $E\left\{D_{\mathrm{s}+\mathrm{c}, k}\right\}$ of all source nodes and the aggregation of the received powers from both the source and the relay nodes is minimized, i.e.

$$
\min _{R_{\mathrm{s}}, R_{\mathrm{c}}, S^{\mathrm{rec}}}\left(\gamma \sum_{k=1}^{K} w_{k} E\left\{D_{\mathrm{s}+\mathrm{c}, k}\right\}+\delta \sum_{n=1}^{K+M} S_{n}^{\mathrm{rec}}\right)
$$

where $w_{k}$ is a weighting factor for the aggregated distortion, and $\gamma, \delta$ are weighting factors with $\gamma+\delta=1$. The weighting factors $(\gamma, \delta)$ indicate the tradeoff among the two formulated objectives, i.e. the enhancement of video quality versus the minimization of the transmission power consumption. The problem is solved under the consideration of the constraints explained in Section II that all interfering nodes transmit using the same bit rate and that each relay node $m$ uses a sufficient bit rate for the simultaneous forwarding of the received video data (Eq. (4)).

\section{A. Definition of Weights for the Aggregation of Distortion}

Using different weights $w_{k}$ for the aggregation of the endto-end video distortion of all source nodes (see Eq. (10)), we can favor different source nodes. Thus, the resources are 
allocated so that nodes with higher weights can deliver videos with enhanced end-to-end video quality. We consider the following different cases:

1) Using Equal Weights for the Aggregation of Distortion $(E W A D)$ : We assume that all source nodes have equal weights, i.e. $w_{k}=1$, which means that their video quality enhancement is of equal importance.

2) Using Motion-related Weights for the Aggregation of Distortion (MWAD): The weights are motion-related, since they are tuned according to parameters $\alpha_{k}$, which reflect the motion level of each recorded video. The weight for each source node $k$ is:

$$
w_{k}=\frac{\alpha_{k}}{\sum_{i=1}^{K} \alpha_{i}}
$$

given that $\sum_{k=1}^{K} w_{k}=1$. In particular, high motion nodes have a higher priority in the minimization of their distortion, and thus, in the enhancement of the delivered video quality.

\section{B. Optimization Algorithm}

In the proposed scheme, the received and transmitted powers are assumed to take continuous values within a specified range, whereas the source and channel coding rates can only have discrete values. As the formulated multi-variable optimization problems are mixed-integer problems, a stochastic optimization technique is selected, called Particle Swarm Optimization (PSO) [14]. PSO is an efficient and adjustable populationbased optimization algorithm that was inspired by social behavior of a colony, e.g. a flock of birds. This technique actually mimics the behavior of a population, the swarm, that consists of a number of individuals, the particles. The swarm has a fixed size of particles that search for the function minimum in a multidimensional space.

\section{Simulation Results}

\section{A. Simulation Settings}

We assume that neighboring visual sensors monitor the same area. Due to this assumption, the neighboring nodes are organized with respect to their location in clusters. We consider a WVSN topology similar to the example of Fig. 1. We assume that 20 source nodes are organized in four clusters of the same cardinality. Taking into account that the BS is out of the transmission range of the source nodes, a relay node is committed to each cluster in order to channel-decode-andforward the video data to the BS. The enumeration of the relays corresponds to the enumeration of the cluster they are committed to (e.g. relay node 1 forwards the video data from cluster 1). Interference exists among the source nodes within a cluster as they transmit their videos to their corresponding relay node. Moreover, the four relay nodes interfere with each other when they retransmit videos to the BS.

Since the five source nodes of each cluster monitor the same area, we assume that they transmit the same video sequences, thus the $\left(\alpha_{k}, \beta_{k}\right)$ parameters of nodes in a cluster are assumed to be equal and invariant in time. In order to evaluate the performance of our method, several cases with different motion levels per cluster have been considered. In the presented results, cluster 1 nodes transmit high motion videos while the nodes of cluster 2 transmit low motion videos and the nodes of clusters 3 and 4 transmit different medium motion videos. The notions "low", "medium" and "high" motion are used for video sequences of similar motion levels with the "Akiyo", "Salesman" and "Foreman" QCIF video sequences of $15 \mathrm{fps}$, respectively.

The range of $[0.100,0.500] \mathrm{W}$ is used for the transmission powers of all source nodes and the range $[0.100,5.000] \mathrm{W}$ is used for the relay nodes. For all links, the total bandwidth $W_{t}$ is $5 \mathrm{MHz}$. The background noise $N_{0}$ is equal to $1 \mathrm{pW} / \mathrm{Hz}$, although various levels of power spectral density of background noise $N_{0}$ have also been tested, providing similar results. For the source nodes in clusters, the set of possible source and channel coding rate choices is $\{(32 \mathrm{kbps}, 1 / 3),(48 \mathrm{kbps}, 1 / 2),(64 \mathrm{kbps}, 2 / 3)\}$, and the total transmission bit rate $R_{k}$ is the same for all cluster nodes and equal to $96 \mathrm{kbps}$. For the relay nodes, the transmission bit rate $R_{m}$ is the same for all and equal to $480 \mathrm{kbps}$. The channel coding rates for the relay nodes are selected from the set $\{1 / 3,1 / 2,2 / 3\}$. RCPC codes with mother rate $1 / 4$ are used.

As far as the values of $(\gamma, \delta)$ are concerned, we have considered the range $[0.50,1.00]$ for $\gamma$ and $[0.00,0.50]$ for $\delta$. In order to reduce the infinite number of points in these ranges, we assumed that $\gamma$ and $\delta$ can take values within the following sets (using a step size equal to 0.05): $\gamma \in\{0.50,0.55, \ldots, 0.95,1.00\} \subset[0.50,1.00]$ and $\delta \in$ $\{0.00,0.05, \ldots, 0.45,0.50\} \subset[0.00,0.50]$, so that $\gamma+\delta=1$. The conducted experiments per case (a number of 30 independent experiments) demonstrate that PSO optimization performs efficiently for the number of problem parameters to be determined (using a number of swarm particles equal to 80 and a maximum number of PSO iterations for convergence equal to 1200).

\section{B. Results and Discussion}

The allocated source and channel coding rates for each cluster as well as the channel coding rates for the relay nodes for the different values of $(\gamma, \delta)$ are reported in Table I. Regarding the resulting channel coding rates for the relay nodes, the weakest channel coding rate has been selected in all cases for all relays, i.e. $2 / 3$. Furthermore, we observe that in all cases, using the highest source coding rate is preferred for the high motion source nodes. On the other hand, for the nodes of medium and low amount of motion, stronger channel coding rate is employed.

For the video quality assessment we use the PSNR that is directly related to the expected video distortion $E\left\{D_{\mathrm{s}+\mathrm{c}}\right\}$, i.e.

$$
P S N R=10 * \log _{10} \frac{255^{2}}{E\left\{D_{\mathrm{s}+\mathrm{c}}\right\}} .
$$


TABLE I

SOURCE AND CHANNEl CODING RATES PER CLUSTER AND RELAY FOR THE VARIOUS VALUES OF $\gamma$.

\begin{tabular}{|c|c|c|c|c|c|c|c|c|}
\hline \multirow[b]{2}{*}{ Cluster } & \multicolumn{4}{|c|}{ EWAD } & \multicolumn{4}{|c|}{ MWAD } \\
\hline & 1 & 2 & 3 & 4 & 1 & 2 & 3 & 4 \\
\hline Motion level & high & low & medium & medium & high & low & medium & medium \\
\hline $\begin{array}{c}\gamma=0.50 \\
\gamma \in[0.55,1.00]\end{array}$ & $\begin{array}{l}\text { (64kbps,2/3) } \\
(64 \mathrm{kbps}, 2 / 3)\end{array}$ & $\begin{array}{l}\text { (48kbps, } 1 / 2) \\
(48 \mathrm{kbps}, 1 / 2)\end{array}$ & $\begin{array}{l}(48 \mathrm{kbps}, 1 / 2) \\
(48 \mathrm{kbps}, 1 / 2)\end{array}$ & $\begin{array}{l}\text { (48kbps, } 1 / 2) \\
(48 \mathrm{kbps}, 1 / 2)\end{array}$ & $\begin{array}{l}\text { (64kbps,2/3) } \\
(64 \mathrm{kbps}, 2 / 3)\end{array}$ & $\begin{array}{l}(32 \mathrm{kbps}, 1 / 3) \\
(32 \mathrm{kbps}, 1 / 3)\end{array}$ & $\begin{array}{l}\text { (32kbps,1/3) } \\
\text { (48kbs, } 1 / 2)\end{array}$ & $\begin{array}{l}\text { (48kbps, } 1 / 2) \\
(48 \mathrm{kbps}, 1 / 2)\end{array}$ \\
\hline Relay & 1 & 2 & 3 & 4 & 1 & 2 & 3 & 4 \\
\hline$\gamma \in[0.50,1.00]$ & $2 / 3$ & $2 / 3$ & $2 / 3$ & $2 / 3$ & $2 / 3$ & $2 / 3$ & $2 / 3$ & $2 / 3$ \\
\hline
\end{tabular}

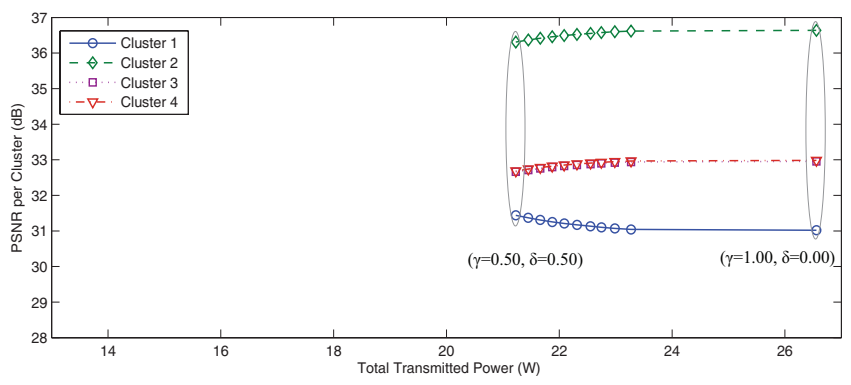

(a) Results for EWAD

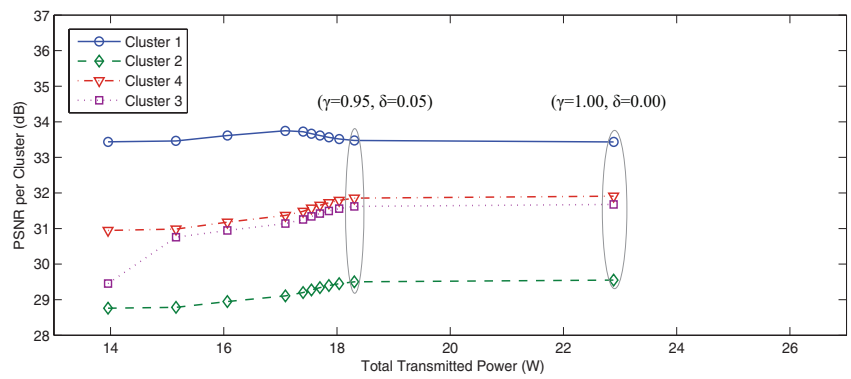

(b) Results for MWAD

Fig. 2. Resulting PSNR per Cluster vs the Total Transmission Power for all $(\gamma, \delta)$ values.

Figure 2 illustrates the resulting video quality in terms of PSNR in respect with the total transmitted power in the considered WVSN for the different values $(\gamma, \delta)$. As anticipated, EWAD favors the low motion nodes in terms of PSNR, while MWAD offers considerably higher PSNR to the high motion nodes for all values of $\gamma$. Using MWAD, we achieve to deliver videos with qualities proportional to their amount of motion.

To better demonstrate the impact of power control on the delivered video quality, we compare the PSNR for $\gamma<1.00$ with the PSNR for $\gamma=1.00$ (when no power control is applied). It is remarkable that using EWAD results in almost the same PSNR for the different $(\gamma, \delta)$ values. Particularly, the highest PSNR difference is $0.4179 \mathrm{~dB}$ and is observed for the high motion nodes (cluster 1) for $\gamma=0.50$. Comparing the PSNR for $\gamma=0.50$ for the nodes of cluster 2 with the PSNR for $\gamma=1.00$, we observe that in order to achieve the highest video quality improvement that is equal to 0.3266 $\mathrm{dB}$, we need to consume $20.24 \%$ more transmission power

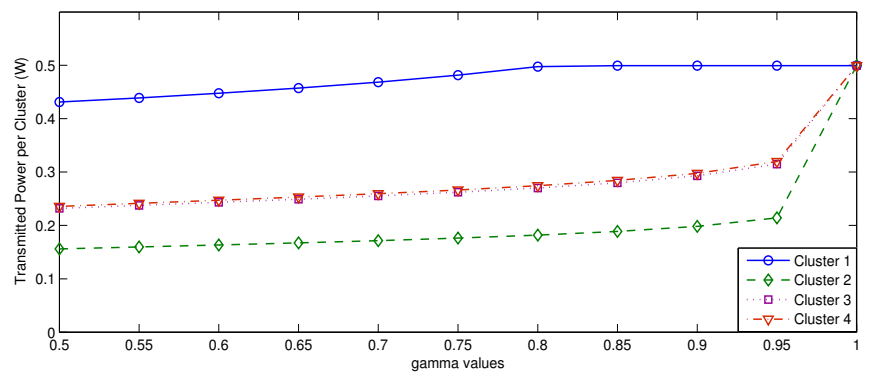

(a) Results for EWAD

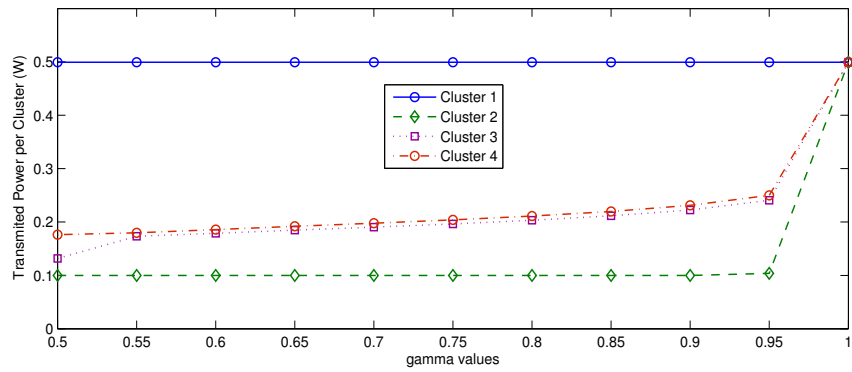

(b) Results for MWAD.

Fig. 3. Transmission Power per Cluster node for the different $\gamma$ values.

in total. Although MWAD achieves lower PSNR values on average, it allocates lower total transmission power compared to EWAD for the same $(\gamma, \delta)$ values, as depicted in Fig. 2(a). It is also important to point out that we can achieve almost the same PSNR for $\gamma=0.95$ and $\gamma=1.00$ (the average PSNR difference for all clusters is $0.0188 \mathrm{~dB}$ ) and at the same time we use $20.01 \%$ less total transmission power (see Fig. 2(b)). Considering these observations, we conclude that when power control is omitted $(\gamma=1.00, \delta=0.00)$, excessive transmission power in total is consumed for a rather small video quality gain.

In Fig. 3, we depict the transmission power per cluster node for the different $\gamma$ values, while in Fig. 4 we illustrate the allocated transmission powers per relay node. In Fig. 3 the effect of power control is clear, since the transmission power increases along with the increase of $\gamma$ value (which means that the weighting factor for power in our problem formulation $\delta$ decreases). Moreover, in the case of $(\gamma=1.00, \delta=0.00)$, when no power control is applied, all source nodes transmit 


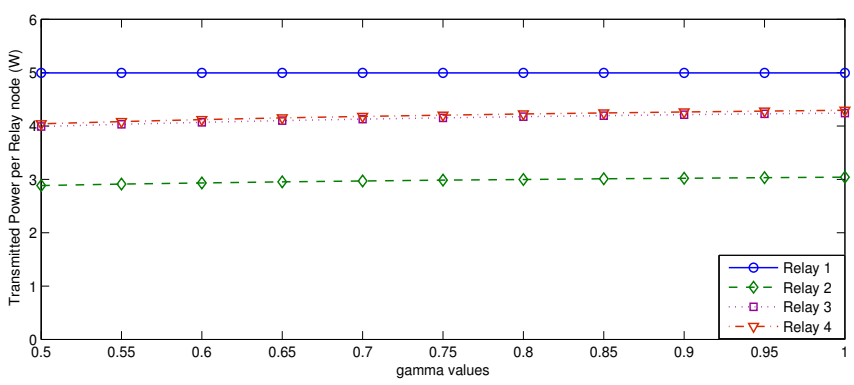

(a) Results for EWAD.

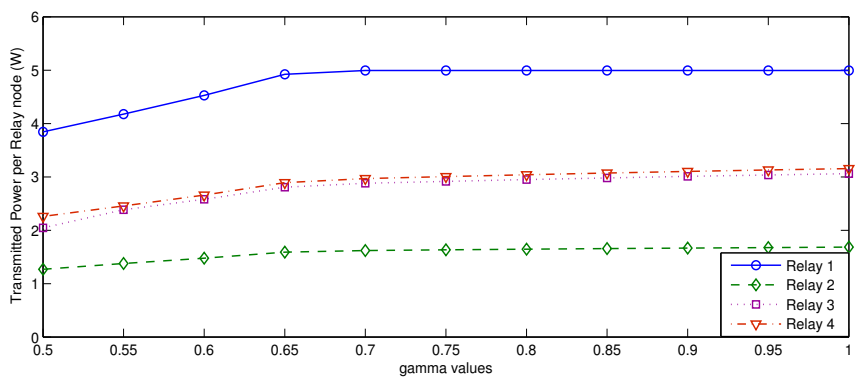

(b) Results for MWAD.

Fig. 4. Transmission Power per Relay node for the different $\gamma$ values.

using the maximum admissible power for both EWAD and MWAD. Besides these, Fig. 3 and Fig. 4 reveal that the allocated transmission powers for each cluster and for each relay are in line with the motion levels of the recorded scenes. However, EWAD assigns higher transmission power than MWAD, especially for the clusters of low and medium motion. For example, using EWAD the transmission power for the low motion nodes for $\gamma<1.00$ ranges from $0.1560 \mathrm{~W}$ to $0.2141 \mathrm{~W}$, whilst using MWAD the range is $0.1000-0.1039 \mathrm{~W}$. This is explained from the fact that using MWAD we intend to favor the clusters in proportion to the amount of motion. So, in order to enhance the video quality of the high motion nodes, MWAD increases their transmission power and at the same time reduces the transmission power of all other clusters and relays. This increases the energy-per-bit to MAI and noise ratio for the high motion nodes and the corresponding relay, while at the same time it reduces for the other clusters and their relays. Hence, the reduction of the transmission power of the low and medium nodes is the main reason of their quality degradation.

Another observation from Fig. 4 is that in the case of EWAD the transmission power increases slightly as the $\gamma$ value increases. Moreover, EWAD assigns higher transmission power than MWAD to all the relay nodes for the different $\gamma$ as well. For example, for relay 3 EWAD assigns on average $1.5 \mathrm{~W}$ higher transmission power than MWAD. Considering this, it is inferred that using MWAD the battery-constrained nodes prolong their lifetime compared to the case that EWAD is utilized.

\section{Conclusions}

In the present paper, we propose a method for effective joint end-to-end video quality enhancement and transmission power control in a multihop DS-CDMA based WVSN. In this bi-objective problem formulation, we use weighting factors that regulate the tradeoff between these two objectives. Furthermore, we define different weights for the aggregation function of the video distortion of the source nodes (EWAD and MWAD), that achieve to favor specific nodes according to the assigned weights. The conducted simulations demonstrate the tradeoff among the delivered video quality and the utilized transmission power. An important conclusion drawn is that excessive transmission power in total is consumed for a rather small video quality gain for certain nodes. Finally, by utilizing MWAD the low and medium motion nodes may experience longer lifetime, while on the other hand the high motion nodes deliver higher video quality.

\section{REFERENCES}

[1] S. Soro and W. Heinzelman, "A Survey of Visual Sensor Networks," in Advances in Multimedia, 2009, pp. 1-22.

[2] U. C. Kozat and I. Koutsopoulos, "Cross-layer design for power efficiency and QoS provisioning in multi-hop wireless networks," IEEE Transactions on Wireless Communications, vol. 5, no. 11, pp. 33063315, November 2006.

[3] R. Devarajan, S. Jha, U. Phuyal, and V. Bhargava, "Energy-aware resource allocation for cooperative cellular network using multi-objective optimization approach," IEEE Transactions on Wireless Communications, vol. PP, no. 99, pp. 1-11, 2012.

[4] Q. Ni and C. C. Zarakovitis, "Nash bargaining game theoretic scheduling for joint channel and power allocation in cognitive radio systems," IEEE Journal on Selected Areas in Communications, vol. 30, no. 1, pp. 70-81, January 2012.

[5] E. G. Datsika, A. V. Katsenou, L. P. Kondi, E. Papapetrou, and K. E. Parsopoulos, "Priority-based cross-layer optimization for multihop DSCDMA visual sensor networks," in Proc. IEEE International Conference on Image Processing, Orlando, Florida, 2012, pp. 1102-1104.

[6] Y. S. Chan and J. W. Modestino, "A joint source coding-power control approach for video transmission over CDMA networks," IEEE Journal on Selected Areas in Communications, vol. 21, no. 10, pp. 1516-1525, 2003.

[7] J. Hagenauer, "Rate-compatible punctured convolutional codes (RCPC codes) and their applications," IEEE Transactions on Communications, vol. 36, no. 4, pp. 389-400, April 1988.

[8] A. Luthra H. Wang, L. P. Kondi and S. Ci, $4 G$ Wireless Video Communications, John Wiley \& Sons, Ltd, 2009.

[9] T. Wiegand and G. Sullivan, "The H.264/AVC Video Coding Standard Standards in a nutshell," IEEE Signal Processing Magazine, vol. 24, no. 2, pp. 148-153, March 2007.

[10] L. P. Kondi and E. S. Bentley, "Game-Theory-based cross-layer optimization for Wireless DS-CDMA Visual Sensor Networks," IEEE International Conference on Image Processing, pp. 4485-4488, 2010.

[11] L. P. Kondi and A. K. Katsaggelos, "Joint source-channel coding for scalable video using models of rate distortion functions," in Proc. IEEE International Conference on Acoustics, Speech and Signal Processing, Salt Lake City, 2001, pp. 1377-1380.

[12] E. S. Bentley, L. P. Kondi, J. D. Matyjas, M. J. Medley, and B. W. Suter, "Spread spectrum visual sensor networks resource management using an end-to-end cross layer design," IEEE Transactions on Multimedia, vol 13 , no. 1, pp. 125-131, February 2011.

[13] T. Rapaport, Wireless Communications Principles and Practice, Prentice Hall Communications Engineering and Emerging Technologies Series. Prentice Hall, 2nd edition, 2001.

[14] K. E. Parsopoulos and M. N. Vrahatis, Particle Swarm Optimization and Intelligence: Advances and Applications, Information Science Publishing (IGI Global), 2010. 